\title{
Estimating Cancer Incidence, Prevalence, and the Number of Cancer Patients Treated with Anti-tumor Therapy in 2015 and 2020 - Analysis of the Czech National Cancer Registry
}

\section{Odhady incidence, prevalence a počtu onkologických pacientů léčených protinádorovou terapií v letech 2015 a 2020 - analýza Národního onkologického registru ČR}

\author{
Dusek L. ${ }^{1,2}$, Pavlik T. ${ }^{1,2}$, Majek O. ${ }^{1,2}$, Buchler T. ${ }^{3}$, Muzik J. ${ }^{1,2}$, \\ Maluskova D. ${ }^{1,2}$, Koptikova J. ${ }^{1}$, Bortlicek Z. ${ }^{1,2}$, Abrahamova J. ${ }^{3}$ \\ Institute of Biostatistics and Analyses, Faculty of Medicine and Faculty of Science, Masaryk University, Brno \\ 2 Institute of Health Information and Statistics of the Czech Republic, Prague \\ ${ }^{3}$ Department of Oncology, Thomayer Hospital, Prague
}

\begin{abstract}
Summary
Background: Cancer burden in the Czech population ranks among the highest worldwide, which introduces a strong need for a prospective modelling of cancer incidence and prevalence rates. Moreover, a prediction of number of cancer patients requiring active anti-tumor therapy is also an important issue. This paper presents the stage-specific predictions of cancer incidence and prevalence, and the stage- and region-specific patients requiring active anti-tumor therapy for the most common cancer diagnoses in the Czech Republic for years 2015 and 2020. The stage-specific estimates are also presented with regard to the treatment phase as newly diagnosed patients, patients treated for non-terminal recurrence, and patients treated for terminal recurrence. Patients and Methods: Data of the Czech National Cancer Registry from 1977 to 2011 has been used for the analysis, omitting the records of patients diagnosed as death certificate only or at autopsy. In total, 1,777,775 incidences have been considered for the estimation using a statistical model utilizing solely the population-based cancer registry data. All estimates have been calculated with respect to the changing demographic structure of the Czech population and the clinical stage at diagnosis. Results: Considering year 2011 as the baseline, we predict $89 \%, 15 \%, 31 \%$ and $32 \%$ increase in prostate, colorectal, female breast and lung cancer incidence, respectively, in 2020 resulting in 13,153, 9,368, 8,695, and 8,604 newly diagnosed cancer patients in that year, respectively. Regarding cancer prevalence in 2020, the estimated increase is $140 \%, 40 \%, 51 \%$, and $17 \%$ for prostate, colorectal, female breast and lung cancer, respectively, meaning that more than 100,000 prevalent female breast cancer patients as well as more than 100,000 prevalent prostate cancer patients are expected in the Czech Republic. The estimated numbers of patients requiring active anti-tumor therapy for prostate, colorectal, female breast and lung cancer in the Czech Republic in 2020 are 23,652, 14,006, 14,759 and 8,272; respectively. Conclusions: The analysis documents a serious increase in cancer incidence and prevalence in the Czech Republic in years 2015 and 2020 when compared to the situation in 2011. Regarding the estimated numbers of patients requiring active anti-tumor therapy, the model confirms a continuous increase that must be accounted for in the future planning of health care in the Czech Republic.
\end{abstract}

Key words

Czech National Cancer Registry - neoplasms - prediction - incidence - prevalence - anticancer therapy
Validation of the Czech National Cancer Registry and population-based monitoring of cancer disparities are supported by grant of the Internal Grant Agency of the Czech Ministry of Health No. NT/13660-4. Models of long-term predictions of cancer therapeutic burden were developed as a part of projects CORIS and PET-CZ(Q), building predictive information infrastructure of the Czech cancer care. The authors greatly acknowledge professional support of data managers of the Czech National Cancer Registry.

Validace dat Národního onkologického registru ČR a populační monitoring rozdílú v onkologické péći jsou podporovány grantem Interní grantové agentury Ministerstva zdravotnictví Č́ č. NT/13660-4. Modely dlouhodobých predikcí léčebné zátěže zhoubnými nádory byly vyvinuty jako součást projektů CORIS a PET-CZ(Q), s jejichž pomocí je budována infrastruktura predikčního systému pro českou onkologickou péči. Autoři vysoce oceňují profesionální podporu ze strany datových manažerů Národního onkologického registru ČR.

The authors declare they have no potential conflicts of interest concerning drugs, products, or services used in the study.

Autoři deklarují, že $v$ souvislosti $s$ predmětem studie nemaji žádné komerčni zájmy.

The Editorial Board declares that the manuscript met the ICMJE "uniform requirements" for biomedical papers.

Redakční rada potvrzuje, že rukopis práce splnil ICMJE kritéria pro publikace zasílané do biomedicínských časopisů.

Assoc. prof. Ladislav Dusek, PhD Institute of Biostatistics and Analyses

Faculty of Medicine and Faculty of Science

Masaryk University

Kamenice 126/3

62500 Brno

Czech Republic

e-mail: dusek@iba.muni.cz

Submitted/Obdrženo: 8. 10. 2014 Accepted/Přijato: 10. 1. 2015

http://dx.doi.org/10.14735/amko201530 


\section{Souhrn}

Východiska: Česká republika se celosvětově řadí mezi země s největším zastoupením onkologických pacientů, což v důsledku vede k potřebě prospektivního modelování epidemiologie nádorových onemocnění, jako je incidence, prevalence a počet pravděpodobně léčených pacientů. Tento článek prezentuje odhady incidence, prevalence a počty pacientů pravděpodobně léčených pro nejčastější nádorová onemocnění $v$ ČR pro roky 2015 a 2020. Odhady jsou prezentovány po stadiích a dále dle jednotlivých fází léčby pacienta v podobě počtu nově diagnostikovaných pacientů, pacientů s progresí/relapsem v neterminální fázi a pacientů v terminální fázi léčby. Pacienti a metody: Analýza byla provedena na datech Národního onkologického registru z období 1977-2011 s vyloučením záznamů diagnostikovaných z listu o prohlídce zemřelého a při pitvě. Celkem jsme uvažovali více než 1,7 milionu záznamů onkologických pacientů. Statistický model kalkuloval odhady incidence, prevalence a počtu pravděpodobně léčených pacientů pouze s využitím populačních dat a s ohledem na měnící se demografickou strukturu populace ČR a klinické stadium nádoru při diagnóze. Výsledky: S ohledem na nejčastější onkologické diagnózy, naše analýza predikuje 89\%, 15\%, 31\% a 32\% nárůst v incidenci zhoubného novotvaru (ZN) prostaty, tlustého střeva a konečníku, prsu u žen, resp. plic v ČR v roce 2020 vzhledem k datům za rok 2011 , což u těchto diagnóz představuje predikovanou incidenci ve výši 13 153, 9368,8695 , resp. 8604 nově diagnostikovaných pacientů. V prevalenci v roce 2020 (opět vztaženo k roku 2011) pak predikujeme pro ZN prostaty, tlustého střeva a konečníku, prsu u žen a plic nárůst ve výši $140 \%$, $40 \%, 51 \%$, resp. $17 \%$, což v případě ZN prostaty a ZN prsu u žen znamená překročení hranice 100000 onkologických pacientů s historií daného onemocnění. Prediktivní odhady počtu pravděpodobně léčených pacientů se ZN prostaty, tlustého střeva a konečníku, prsu u žen a plic v ČR pro rok 2020 jsou 23 652, 14 006, 14 759, resp. 8 272. Závěr: Naše analýza potvrzuje výrazné zvýšení incidence a prevalence nádorových onemocnění v ČR v letech 2015 a 2020 a u vybraných diagnóz prezentuje počty pacientů, kteří budou v letech 2015 a 2020 pravděpodobně vyžadovat protinádorovou léčbu. Tyto odhady mohou sloužit k analýze rizik, monitorování kvality léčby a k optimalizaci ekonomických nákladů souvisejících s onkologickou péčí.

\section{Klíčová slova}

Národní onkologický registr - zhoubné nádory - predikce - incidence - prevalence - protinádorová léčba

\section{Background}

Cancer burden in the Czech population ranks among the highest worldwide and has been growing continuously $[1,2]$. During 1990s and 2000s, the incidence of all major cancers was constantly increasing in the Czech population [3], and the growth dynamic was consistent with recently published international data $[1,4-6]$. The number of cancer patients is very likely to grow further, as a result of the ageing population. If health care is to be assessed quantitatively, knowing the number of cancer patients is an essential condition and a fundamental input. Thus, there is a strong need for a prospective modelling of cancer incidence and prevalence rates, as these measures are necessary for monitoring of the overall cancer load and its dynamics [7]. Moreover, a prediction of number of cancer patients requiring active anti-tumor therapy is an important issue, as necessary financial resources need to be allocated. Due to centralized and decentralized part of cancer care, region-specific estimates are of a great importance as well. The disease extent should be also taken into account in the model as the clinical stage is, regarding patient life-expectation and anticipated financial costs, even more important than age [8]. However, the effort to estimate cancer prevalence on a stage-specific basis is a challenging task $[9,10]$. The stage-specific modelling is complicated and requires a comprehensive approach, since the stage at the time of diagnosis need not necessarily coincide with the disease extent several years afterwards.

This paper presents the stage-specific predictions of cancer incidence and prevalence, and the stage- and region-specific estimates of patients requiring active anti-tumor therapy for the common diagnostic groups of malignant tumors in the Czech Republic for years 2015 and 2020. Statistical model utilizing solely the population-based cancer registry data was used for this purpose [10]. The estimated numbers of probably treated patients are given with respect to individual phases of the anti-tumor therapy - the number of newly diagnosed and treated patients, the number of patients treated for non-terminal cancer recurrence and the number of patients treated for terminal cancer recurrence.

\section{Patients and Methods}

\section{Patients}

Data of the Czech National Cancer Registry (CNCR), which was established in 1976 as a nationwide registry
$(10,230,000$ inhabitants according to the 2001 census), was used for the analysis. Until the end of 2011, there were more than 1.9 million cancer cases recorded in the CNCR. Malignant neoplasms were recorded according to the International statistical classification of diseases and related health problems, $10^{\text {th }}$ revision (ICD-10) [11], and International Classification of Diseases for Oncology (ICD-O, $3^{\text {rd }}$ revision) [12]. Tumors were staged on the basis of TNM classification system, currently $7^{\text {th }}$ edition [13].

Only clinically relevant cancer records entered the modelling procedures. In some cancer diagnoses, data on cases diagnosed in 1977-1981 were excluded due to the lack of classification system for clinical stages. Moreover, the records on patients diagnosed as death certificate only or at autopsy were excluded from the analysis as well. Finally, 1,777,775 incident cases were considered for analysis.

Four age categories were considered in the estimation process - 15-49 years (children under 15 years were included only among brain cancer, Hodgkin and non-Hodgkin lymphoma patients), $50-64$ years, $65-79$ years and $80+$ years; as well as four categories for the disease extent - clinical stages I, II, III, and IV. In some cancers, however, stages I and II 
were merged prior to analyses due to changes in the TNM classification system in 1995 [14]. Moreover, cases with missing information on stage (denoted as X) were also considered for the model, as they represent an indispensable mass of all records.

\section{Methods}

The model is based on data from the $\mathrm{CNCR}$, and all estimates have been done with respect to the changing demographic structure of the Czech population. The concept of estimation comes from the model of time interval prevalence, a number of patients who both have a present or past diagnosis of cancer and are alive in a population during a certain period. Detailed description of the model was published elsewhere [10]. Regarding one age group, the stage-specific prevalence in calendar year y can be expressed as the convolution of the stage-specific incidence at i years prior to the calendar year $y$ and the corresponding i-year survival rate. To obtain the number of patients receiving active anti-tumor therapy, the stage-specific prevalence needs to be further corrected for the probability of being untreated with anti-tumor therapy due to poor health condition or other objective reasons, and, simultaneously, it needs to be corrected in a way that the only considered patients are those with the recurrence of the disease in a good health condition to allow for anti-tumor treatment. The correction is accomplished using the stage-specific probability of being treated with an anti-tumor treatment in the year $y$ and the function that describes the probability of cancer recurrence after surviving i years after primary diagnosis. Moreover, we assume that each patient diagnosed in stage $s$ can suffer in time from two forms of cancer recurrence, either non-terminal (actually not leading to death in the year y), or terminal (leading to death in the year y).

The individual components of the model are then estimated as follows:

- In view of the fact that changes in incidence rates of malignant tumors very frequently reflect changes in the

Tab. 1. Cancer epidemiology in the Czech Republic (all cancers including skin neoplasms C00-C97, data from 2011).

\begin{tabular}{|c|c|c|c|}
\hline $\begin{array}{l}\text { Parameter } \\
\text { overall incidence }\end{array}$ & Males & Females & Total \\
\hline absolute number & 41,349 & 36,424 & 77,773 \\
\hline rate per 100,000 & 801.6 & 681.2 & 740.3 \\
\hline \multicolumn{4}{|l|}{ overall mortality ${ }^{1}$} \\
\hline absolute number & 15,008 & 12,163 & 27,171 \\
\hline $\begin{array}{l}\text { cases per } 100,000 \\
\text { population }\end{array}$ & 291.0 & 227.5 & 258.6 \\
\hline
\end{tabular}

\section{prevalence}

\begin{tabular}{|c|c|c|c|}
\hline absolute number & 206,969 & 268,493 & 475,462 \\
\hline cases per 100,000 & 4,012 & 5,021 & 4,526 \\
\hline
\end{tabular}

population

\section{growth index (2001-2011)}

\begin{tabular}{|l|l|l|l|}
\hline incidence & $32.0 \%$ & $22.8 \%$ & $27.6 \%$ \\
\hline mortality & $-4.7 \%$ & $-5.6 \%$ & $-5.0 \%$ \\
\hline prevalence & $62.4 \%$ & $50.0 \%$ & $55.0 \%$ \\
\hline
\end{tabular}

${ }^{1}$ mortality statistics - Czech Statistical Office

distribution of age categories in the monitored population, the predictions of incidence rates take into account information on developments in age structure of the Czech population. The calculation is based on the Poisson regression model. Point estimates are supplemented with $90 \%$ Cls, using the delta-method [15] to estimate the standard error.

- The life-table method [16] using the moving window principle is employed as a method to estimate the overall stage-specific survival rates. In this procedure, the survival rates are estimated successively, using the cohort analysis of patients diagnosed in partially overlapping 5-year time intervals.

- Regarding non-terminal cancer recurrence, it is estimated using the information on the patient's health status and anti-tumor therapy applied after the first year following diagnosis (first year after diagnosis is assumed to correspond to the initial treatment phase [17]). As for terminal cancer recurrence, the approach is based on the simplifying assumption that nobody can die from cancer, with cancer being the main reason of death, without passing through the phase of generalized disease.

- The proportion of patients treated with anti-tumor therapy reflecting the patients' health status is derived from the CNCR population data on primary anti-tumor therapy.

Four scenarios combining progress in incidence rates (modelled or stabilized) and survival rates (improving or stabilized) were considered in the prevalence model. However, results corresponding to only one of the scenarios are presented for each diagnosis due to space restrictions. The estimated numbers of probably treated cancer patient are subsequently localized for regions of the Czech Republic, using the same methodical approaches. In particular, these partial 
Tab. 2. Predicted values of cancer incidence in the Czech Republic in 2015 according to diagnosis and clinical stage of primary tumor. Numbers of cases are accompanied with $90 \%$ confidence intervals (in brackets).

\begin{tabular}{|c|c|c|c|c|c|c|}
\hline Diagnosis (ICD-10 code) & Stage I & Stage II & Stage III & Stage IV & $\begin{array}{l}\text { Stage } \\
\text { unknown }\end{array}$ & Total \\
\hline $\begin{array}{l}\text { head and neck } \\
(\mathrm{C} 00-\mathrm{C} 14, \mathrm{C} 30-\mathrm{C} 31)\end{array}$ & $\begin{array}{c}210 \\
(162 ; 260)\end{array}$ & $\begin{array}{c}158 \\
(128 ; 187)\end{array}$ & $\begin{array}{c}279 \\
(229 ; 329)\end{array}$ & $\begin{array}{c}809 \\
(727 ; 891)\end{array}$ & $\begin{array}{c}183 \\
(133 ; 232)\end{array}$ & $\begin{array}{c}1,639 \\
(1,379 ; 1,899)\end{array}$ \\
\hline $\begin{array}{l}\text { oesophagus } \\
\text { (C15) }\end{array}$ & $\begin{array}{c}39 \\
(28 ; 51)\end{array}$ & $\begin{array}{c}181 \\
(150 ; 213)\end{array}$ & $\begin{array}{c}208 \\
(174 ; 242)\end{array}$ & $\begin{array}{c}297 \\
(259 ; 335)\end{array}$ & $\begin{array}{c}42 \\
(24 ; 60)\end{array}$ & $\begin{array}{c}767 \\
(635 ; 901)\end{array}$ \\
\hline $\begin{array}{l}\text { stomach } \\
\text { (C16) }\end{array}$ & $\begin{array}{c}360 \\
(312 ; 408)\end{array}$ & $\begin{array}{c}165 \\
(126 ; 203)\end{array}$ & $\begin{array}{c}230 \\
(173 ; 287)\end{array}$ & $\begin{array}{c}776 \\
(684 ; 869)\end{array}$ & $\begin{array}{c}138 \\
(81 ; 197)\end{array}$ & $\begin{array}{c}1,669 \\
(1,376 ; 1,964)\end{array}$ \\
\hline $\begin{array}{l}\text { colorectal cancer } \\
\text { (C18-C20) }\end{array}$ & $\begin{array}{c}2,138 \\
(1,973 ; 2,302)\end{array}$ & $\begin{array}{c}1,958 \\
(1,847 ; 2,069)\end{array}$ & $\begin{array}{c}2,042 \\
(1,893 ; 2,190)\end{array}$ & $\begin{array}{c}2,195 \\
(2,083 ; 2,309)\end{array}$ & $\begin{array}{c}245 \\
(169 ; 320)\end{array}$ & $\begin{array}{c}8,578 \\
(7,965 ; 9,190)\end{array}$ \\
\hline $\begin{array}{l}\text { pancreas } \\
\text { (C25) }\end{array}$ & \multicolumn{2}{|c|}{$\begin{array}{c}566 \\
(456 ; 678)\end{array}$} & $\begin{array}{c}188 \\
(120 ; 256)\end{array}$ & $\begin{array}{c}1,312 \\
(1,086 ; 1,540)\end{array}$ & $\begin{array}{c}279 \\
(109 ; 449)\end{array}$ & $\begin{array}{c}2,345 \\
(1,771 ; 2,923)\end{array}$ \\
\hline $\begin{array}{l}\operatorname{larynx} \\
\text { (C32) }\end{array}$ & $\begin{array}{c}140 \\
(104 ; 177)\end{array}$ & $\begin{array}{c}93 \\
(74 ; 112)\end{array}$ & $\begin{array}{c}122 \\
(96 ; 148)\end{array}$ & $\begin{array}{c}188 \\
(155 ; 221)\end{array}$ & $\begin{array}{c}15 \\
(4 ; 26)\end{array}$ & $\begin{array}{c}558 \\
(433 ; 684)\end{array}$ \\
\hline $\begin{array}{l}\text { lung } \\
\text { (C34) }\end{array}$ & $\begin{array}{c}769 \\
(700 ; 837)\end{array}$ & $\begin{array}{c}338 \\
(295 ; 380)\end{array}$ & $\begin{array}{c}1,618 \\
(1,439 ; 1,796)\end{array}$ & $\begin{array}{c}4,201 \\
(3,960 ; 4,442)\end{array}$ & $\begin{array}{c}598 \\
(369 ; 826)\end{array}$ & $\begin{array}{c}7,524 \\
(6,763 ; 8,281)\end{array}$ \\
\hline $\begin{array}{l}\text { melanoma of skin } \\
\text { (C43) }\end{array}$ & $\begin{array}{c}1,835 \\
(1,680 ; 1,991)\end{array}$ & $\begin{array}{c}418 \\
(364 ; 471)\end{array}$ & $\begin{array}{c}119 \\
(85 ; 152)\end{array}$ & $\begin{array}{c}105 \\
(85 ; 123)\end{array}$ & $\begin{array}{c}48 \\
(29 ; 67)\end{array}$ & $\begin{array}{c}2,525 \\
(2,243 ; 2,804)\end{array}$ \\
\hline $\begin{array}{l}\text { female breast } \\
\text { (C50) }\end{array}$ & $\begin{array}{c}3,448 \\
(3,113 ; 3,782)\end{array}$ & $\begin{array}{c}2,264 \\
(2,068 ; 2,461)\end{array}$ & $\begin{array}{c}1,179 \\
(1,052 ; 1,307)\end{array}$ & $\begin{array}{c}528 \\
(452 ; 606)\end{array}$ & $\begin{array}{c}88 \\
(46 ; 129)\end{array}$ & $\begin{array}{c}7,507 \\
(6,731 ; 8,285)\end{array}$ \\
\hline $\begin{array}{l}\text { cervix uteri } \\
\text { (C53) }\end{array}$ & $\begin{array}{c}511 \\
(454 ; 567)\end{array}$ & $\begin{array}{c}116 \\
(84 ; 148)\end{array}$ & $\begin{array}{c}213 \\
(177 ; 249)\end{array}$ & $\begin{array}{c}156 \\
(125 ; 187)\end{array}$ & $\begin{array}{c}29 \\
(17 ; 41)\end{array}$ & $\begin{array}{c}1,025 \\
(857 ; 1,192)\end{array}$ \\
\hline $\begin{array}{l}\text { uterus } \\
\text { (C54) }\end{array}$ & $\begin{array}{c}1,315 \\
(1,200 ; 1,430)\end{array}$ & $\begin{array}{c}213 \\
(168 ; 259)\end{array}$ & $\begin{array}{c}237 \\
(187 ; 287)\end{array}$ & $\begin{array}{c}102 \\
(77 ; 128)\end{array}$ & $\begin{array}{c}212 \\
(138 ; 286)\end{array}$ & $\begin{array}{c}2,079 \\
(1,770 ; 2,390)\end{array}$ \\
\hline $\begin{array}{l}\text { ovary } \\
\text { (C56) }\end{array}$ & $\begin{array}{c}208 \\
(170 ; 248)\end{array}$ & $\begin{array}{c}90 \\
(69 ; 112)\end{array}$ & $\begin{array}{c}420 \\
(375 ; 463)\end{array}$ & $\begin{array}{c}351 \\
(311 ; 390)\end{array}$ & $\begin{array}{c}60 \\
(38 ; 82)\end{array}$ & $\begin{array}{c}1,129 \\
(963 ; 1,295)\end{array}$ \\
\hline $\begin{array}{l}\text { prostate } \\
\text { (C61) }\end{array}$ & \multicolumn{2}{|c|}{$\begin{array}{c}7,147 \\
(6,704 ; 7,590)\end{array}$} & $\begin{array}{c}1,279 \\
(1,165 ; 1,392)\end{array}$ & $\begin{array}{c}1,082 \\
(1,015 ; 1,150)\end{array}$ & $\begin{array}{c}185 \\
(88 ; 282)\end{array}$ & $\begin{array}{c}9,693 \\
(8,972 ; 10,414)\end{array}$ \\
\hline $\begin{array}{l}\text { testis } \\
\text { (C62) }\end{array}$ & $\begin{array}{c}370 \\
(316 ; 425)\end{array}$ & $\begin{array}{c}84 \\
(48 ; 120)\end{array}$ & $\begin{array}{c}62 \\
(38 ; 86)\end{array}$ & * & $\begin{array}{c}15 \\
(3 ; 27)\end{array}$ & $\begin{array}{c}531 \\
(405 ; 658)\end{array}$ \\
\hline $\begin{array}{l}\text { kidney } \\
\text { (C64) }\end{array}$ & \multicolumn{2}{|c|}{$\begin{array}{c}2,075 \\
(1,919 ; 2,231)\end{array}$} & $\begin{array}{c}364 \\
(317 ; 412)\end{array}$ & $\begin{array}{c}658 \\
(567 ; 749)\end{array}$ & $\begin{array}{c}241 \\
(179 ; 304)\end{array}$ & $\begin{array}{c}3,338 \\
(2,982 ; 3,696)\end{array}$ \\
\hline $\begin{array}{l}\text { bladder } \\
\text { (C67) }\end{array}$ & $\begin{array}{c}1,681 \\
(1,525 ; 1,838)\end{array}$ & $\begin{array}{c}458 \\
(407 ; 509)\end{array}$ & $\begin{array}{c}148 \\
(116 ; 181)\end{array}$ & $\begin{array}{c}341 \\
(278 ; 404)\end{array}$ & $\begin{array}{c}50 \\
(24 ; 76)\end{array}$ & $\begin{array}{c}2,678 \\
(2,350 ; 3,008)\end{array}$ \\
\hline $\begin{array}{l}\text { brain } \\
\text { (C71) }\end{array}$ & $* *$ & $* *$ & $* *$ & $* *$ & ** & $\begin{array}{c}915 \\
(804 ; 1,028)\end{array}$ \\
\hline $\begin{array}{l}\text { thyroid } \\
\text { (C73) }\end{array}$ & $\begin{array}{c}900 \\
(828 ; 972)\end{array}$ & $\begin{array}{c}59 \\
(38 ; 80)\end{array}$ & $\begin{array}{c}107 \\
(85 ; 129)\end{array}$ & $\begin{array}{c}123 \\
(95 ; 149)\end{array}$ & $\begin{array}{c}139 \\
(85 ; 192)\end{array}$ & $\begin{array}{c}1,328 \\
(1,131 ; 1,522)\end{array}$ \\
\hline $\begin{array}{l}\text { Hodgkin lymphoma } \\
\text { (C81) }\end{array}$ & $\begin{array}{c}35 \\
(19 ; 51)\end{array}$ & $\begin{array}{c}115 \\
(80 ; 150)\end{array}$ & $\begin{array}{c}69 \\
(49 ; 88)\end{array}$ & $\begin{array}{c}32 \\
(18 ; 47)\end{array}$ & $\begin{array}{c}25 \\
(13 ; 38)\end{array}$ & $\begin{array}{c}276 \\
(179 ; 374)\end{array}$ \\
\hline $\begin{array}{l}\text { non-Hodgkin lymphoma } \\
\text { (C82-C85) }\end{array}$ & $\begin{array}{c}220 \\
(183 ; 256)\end{array}$ & $\begin{array}{c}382 \\
(331 ; 432)\end{array}$ & $\begin{array}{c}351 \\
(309 ; 393)\end{array}$ & $\begin{array}{c}332 \\
(300 ; 365)\end{array}$ & $\begin{array}{c}272 \\
(215 ; 329)\end{array}$ & $\begin{array}{c}1,557 \\
(1,338 ; 1,775)\end{array}$ \\
\hline $\begin{array}{l}\text { multiple myeloma } \\
\text { (C90) }\end{array}$ & $* *$ & $* *$ & $* *$ & $* *$ & $* *$ & $\begin{array}{c}584 \\
(527 ; 641)\end{array}$ \\
\hline
\end{tabular}


Tab. 3. Predicted values of cancer incidence in the Czech Republic in 2020 according to diagnosis and clinical stage of primary tumor. Numbers of cases are accompanied with $90 \%$ confidence intervals (in brackets).

\begin{tabular}{|c|c|c|c|c|c|c|}
\hline Diagnosis (ICD-10 code) & Stage I & Stage II & Stage III & Stage IV & $\begin{array}{l}\text { Stage } \\
\text { unknown }\end{array}$ & Total \\
\hline $\begin{array}{l}\text { head and neck } \\
(\mathrm{C} 00-\mathrm{C} 14, \mathrm{C} 30-\mathrm{C} 31)\end{array}$ & $\begin{array}{c}223 \\
(157 ; 289)\end{array}$ & $\begin{array}{c}168 \\
(128 ; 208)\end{array}$ & $\begin{array}{c}327 \\
(260 ; 395)\end{array}$ & $\begin{array}{c}963 \\
(854 ; 1,072)\end{array}$ & $\begin{array}{c}177 \\
(116 ; 238)\end{array}$ & $\begin{array}{c}1,858 \\
(1,515 ; 2,202)\end{array}$ \\
\hline $\begin{array}{l}\text { oesophagus } \\
\text { (C15) }\end{array}$ & $\begin{array}{c}51 \\
(35 ; 68)\end{array}$ & $\begin{array}{c}236 \\
(194 ; 278)\end{array}$ & $\begin{array}{c}265 \\
(218 ; 312)\end{array}$ & $\begin{array}{c}384 \\
(333 ; 436)\end{array}$ & $\begin{array}{c}25 \\
(10 ; 40)\end{array}$ & $\begin{array}{c}961 \\
(790 ; 1,134)\end{array}$ \\
\hline $\begin{array}{l}\text { stomach } \\
\text { (C16) }\end{array}$ & $\begin{array}{c}428 \\
(359 ; 498)\end{array}$ & $\begin{array}{c}167 \\
(113 ; 219)\end{array}$ & $\begin{array}{c}239 \\
(160 ; 318)\end{array}$ & $\begin{array}{c}880 \\
(750 ; 1,010)\end{array}$ & $\begin{array}{c}85 \\
(37 ; 133)\end{array}$ & $\begin{array}{c}1,799 \\
(1,419 ; 2,178)\end{array}$ \\
\hline $\begin{array}{l}\text { colorectal cancer } \\
\text { (C18-C20) }\end{array}$ & $\begin{array}{c}2,556 \\
(2,316 ; 2,796)\end{array}$ & $\begin{array}{c}2,023 \\
(1,872 ; 2,175)\end{array}$ & $\begin{array}{c}2,236 \\
(2,073 ; 2,398)\end{array}$ & $\begin{array}{c}2,416 \\
(2,293 ; 2,538)\end{array}$ & $\begin{array}{c}137 \\
(80 ; 193)\end{array}$ & $\begin{array}{c}9,368 \\
(8,634 ; 10,100)\end{array}$ \\
\hline $\begin{array}{l}\text { pancreas } \\
\text { (C25) }\end{array}$ & \multicolumn{2}{|c|}{$\begin{array}{c}740 \\
(599 ; 879)\end{array}$} & $\begin{array}{c}218 \\
(130 ; 305)\end{array}$ & $\begin{array}{c}1,537 \\
(1,244 ; 1,829)\end{array}$ & $\begin{array}{c}190 \\
(37 ; 343)\end{array}$ & $\begin{array}{c}2,685 \\
(2,010 ; 3,356)\end{array}$ \\
\hline $\begin{array}{l}\operatorname{larynx} \\
\text { (C32) }\end{array}$ & $\begin{array}{c}143 \\
(93 ; 194)\end{array}$ & $\begin{array}{c}105 \\
(79 ; 130)\end{array}$ & $\begin{array}{c}132 \\
(97 ; 168)\end{array}$ & $\begin{array}{c}202 \\
(158 ; 247)\end{array}$ & $\begin{array}{c}8 \\
(0 ; 17)\end{array}$ & $\begin{array}{c}590 \\
(427 ; 756)\end{array}$ \\
\hline $\begin{array}{l}\text { lung } \\
\text { (C34) }\end{array}$ & $\begin{array}{c}905 \\
(811 ; 999)\end{array}$ & $\begin{array}{c}327 \\
(275 ; 378)\end{array}$ & $\begin{array}{c}1,752 \\
(1,494 ; 2,009)\end{array}$ & $\begin{array}{c}5,210 \\
(4,874 ; 5,544)\end{array}$ & $\begin{array}{c}410 \\
(205 ; 616)\end{array}$ & $\begin{array}{c}8,604 \\
(7,659 ; 9,546)\end{array}$ \\
\hline $\begin{array}{l}\text { melanoma of skin } \\
\text { (C43) }\end{array}$ & $\begin{array}{c}2,269 \\
(2,059 ; 2,478)\end{array}$ & $\begin{array}{c}508 \\
(436 ; 580)\end{array}$ & $\begin{array}{c}105 \\
(68 ; 142)\end{array}$ & $\begin{array}{c}117 \\
(91 ; 143)\end{array}$ & $\begin{array}{c}36 \\
(17 ; 55)\end{array}$ & $\begin{array}{c}3,035 \\
(2,671 ; 3,398)\end{array}$ \\
\hline $\begin{array}{l}\text { female breast } \\
\text { (C50) }\end{array}$ & $\begin{array}{c}4,227 \\
(3,771 ; 4,682)\end{array}$ & $\begin{array}{c}2,391 \\
(2,142 ; 2,642)\end{array}$ & $\begin{array}{c}1,464 \\
(1,286 ; 1,643)\end{array}$ & $\begin{array}{c}568 \\
(463 ; 674)\end{array}$ & $\begin{array}{c}45 \\
(15 ; 75)\end{array}$ & $\begin{array}{c}8,695 \\
(7,677 ; 9,716)\end{array}$ \\
\hline $\begin{array}{l}\text { cervix uteri } \\
\text { (C53) }\end{array}$ & $\begin{array}{c}524 \\
(453 ; 596)\end{array}$ & $\begin{array}{c}108 \\
(67 ; 148)\end{array}$ & $\begin{array}{c}220 \\
(173 ; 269)\end{array}$ & $\begin{array}{c}190 \\
(149 ; 232)\end{array}$ & $\begin{array}{c}16 \\
(7 ; 25)\end{array}$ & $\begin{array}{c}1,058 \\
(849 ; 1,270)\end{array}$ \\
\hline $\begin{array}{l}\text { uterus } \\
\text { (C54) }\end{array}$ & $\begin{array}{c}1,438 \\
(1,285 ; 1,591)\end{array}$ & $\begin{array}{c}248 \\
(187 ; 308)\end{array}$ & $\begin{array}{c}289 \\
(224 ; 353)\end{array}$ & $\begin{array}{c}122 \\
(92 ; 153)\end{array}$ & $\begin{array}{c}204 \\
(112 ; 296)\end{array}$ & $\begin{array}{c}2,301 \\
(1,900 ; 2,701)\end{array}$ \\
\hline $\begin{array}{l}\text { ovary } \\
\text { (C56) }\end{array}$ & $\begin{array}{c}199 \\
(153 ; 246)\end{array}$ & $\begin{array}{c}99 \\
(70 ; 130)\end{array}$ & $\begin{array}{c}465 \\
(408 ; 522)\end{array}$ & $\begin{array}{c}372 \\
(318 ; 424)\end{array}$ & $\begin{array}{c}38 \\
(18 ; 57)\end{array}$ & $\begin{array}{c}1,173 \\
(967 ; 1,379)\end{array}$ \\
\hline $\begin{array}{l}\text { prostate } \\
\text { (C61) }\end{array}$ & \multicolumn{2}{|c|}{$\begin{array}{c}10,014 \\
(9,401 ; 10,626)\end{array}$} & $\begin{array}{c}1,732 \\
(1,580 ; 1,884)\end{array}$ & $\begin{array}{c}1,312 \\
(1,215 ; 1,409)\end{array}$ & $\begin{array}{c}95 \\
(25 ; 164)\end{array}$ & $\begin{array}{c}13,153 \\
(12,221 ; 14,083)\end{array}$ \\
\hline $\begin{array}{l}\text { testis } \\
\text { (C62) }\end{array}$ & $\begin{array}{c}400 \\
(333 ; 468)\end{array}$ & $\begin{array}{c}87 \\
(43 ; 129)\end{array}$ & $\begin{array}{c}58 \\
(29 ; 86)\end{array}$ & * & $\begin{array}{c}11 \\
(0 ; 22)\end{array}$ & $\begin{array}{c}556 \\
(405 ; 705)\end{array}$ \\
\hline $\begin{array}{l}\text { kidney } \\
\text { (C64) }\end{array}$ & \multicolumn{2}{|c|}{$\begin{array}{c}2,471 \\
(2,253 ; 2,689)\end{array}$} & $\begin{array}{c}418 \\
(352 ; 485)\end{array}$ & $\begin{array}{c}767 \\
(637 ; 896)\end{array}$ & $\begin{array}{c}180 \\
(119 ; 240)\end{array}$ & $\begin{array}{c}3,836 \\
(3,361 ; 4,310)\end{array}$ \\
\hline $\begin{array}{l}\text { bladder } \\
\text { (C67) }\end{array}$ & $\begin{array}{c}2,031 \\
(1,810 ; 2,253)\end{array}$ & $\begin{array}{c}535 \\
(466 ; 605)\end{array}$ & $\begin{array}{c}162 \\
(117 ; 206)\end{array}$ & $\begin{array}{c}454 \\
(365 ; 544)\end{array}$ & $\begin{array}{c}21 \\
(5 ; 38)\end{array}$ & $\begin{array}{c}3,203 \\
(2,763 ; 3,646)\end{array}$ \\
\hline $\begin{array}{l}\text { brain } \\
\text { (C71) }\end{array}$ & $* *$ & $* *$ & $* *$ & $* *$ & ** & $\begin{array}{c}1,013 \\
(861 ; 1,167)\end{array}$ \\
\hline $\begin{array}{l}\text { thyroid } \\
\text { (C73) }\end{array}$ & $\begin{array}{c}1,165 \\
(1,072 ; 1,258)\end{array}$ & $\begin{array}{c}46 \\
(25 ; 67)\end{array}$ & $\begin{array}{c}135 \\
(105 ; 165)\end{array}$ & $\begin{array}{c}163 \\
(126 ; 200)\end{array}$ & $\begin{array}{c}154 \\
(87 ; 221)\end{array}$ & $\begin{array}{c}1,663 \\
(1,415 ; 1,911)\end{array}$ \\
\hline $\begin{array}{l}\text { Hodgkin lymphoma } \\
\text { (C81) }\end{array}$ & $\begin{array}{c}35 \\
(15 ; 55)\end{array}$ & $\begin{array}{c}128 \\
(84 ; 172)\end{array}$ & $\begin{array}{c}85 \\
(59 ; 110)\end{array}$ & $\begin{array}{c}35 \\
(16 ; 54)\end{array}$ & $\begin{array}{c}16 \\
(6 ; 25)\end{array}$ & $\begin{array}{c}299 \\
(180 ; 416)\end{array}$ \\
\hline $\begin{array}{l}\text { non-Hodgkin lymphoma } \\
\text { (C82-C85) }\end{array}$ & $\begin{array}{c}248 \\
(196 ; 299)\end{array}$ & $\begin{array}{c}519 \\
(449 ; 588)\end{array}$ & $\begin{array}{c}478 \\
(420 ; 534)\end{array}$ & $\begin{array}{c}402 \\
(358 ; 447)\end{array}$ & $\begin{array}{c}216 \\
(157 ; 275)\end{array}$ & $\begin{array}{c}1,863 \\
(1,580 ; 2,143)\end{array}$ \\
\hline $\begin{array}{l}\text { multiple myeloma } \\
\text { (C90) }\end{array}$ & $* *$ & $* *$ & $* *$ & ** & ** & $\begin{array}{c}649 \\
(567 ; 731)\end{array}$ \\
\hline
\end{tabular}




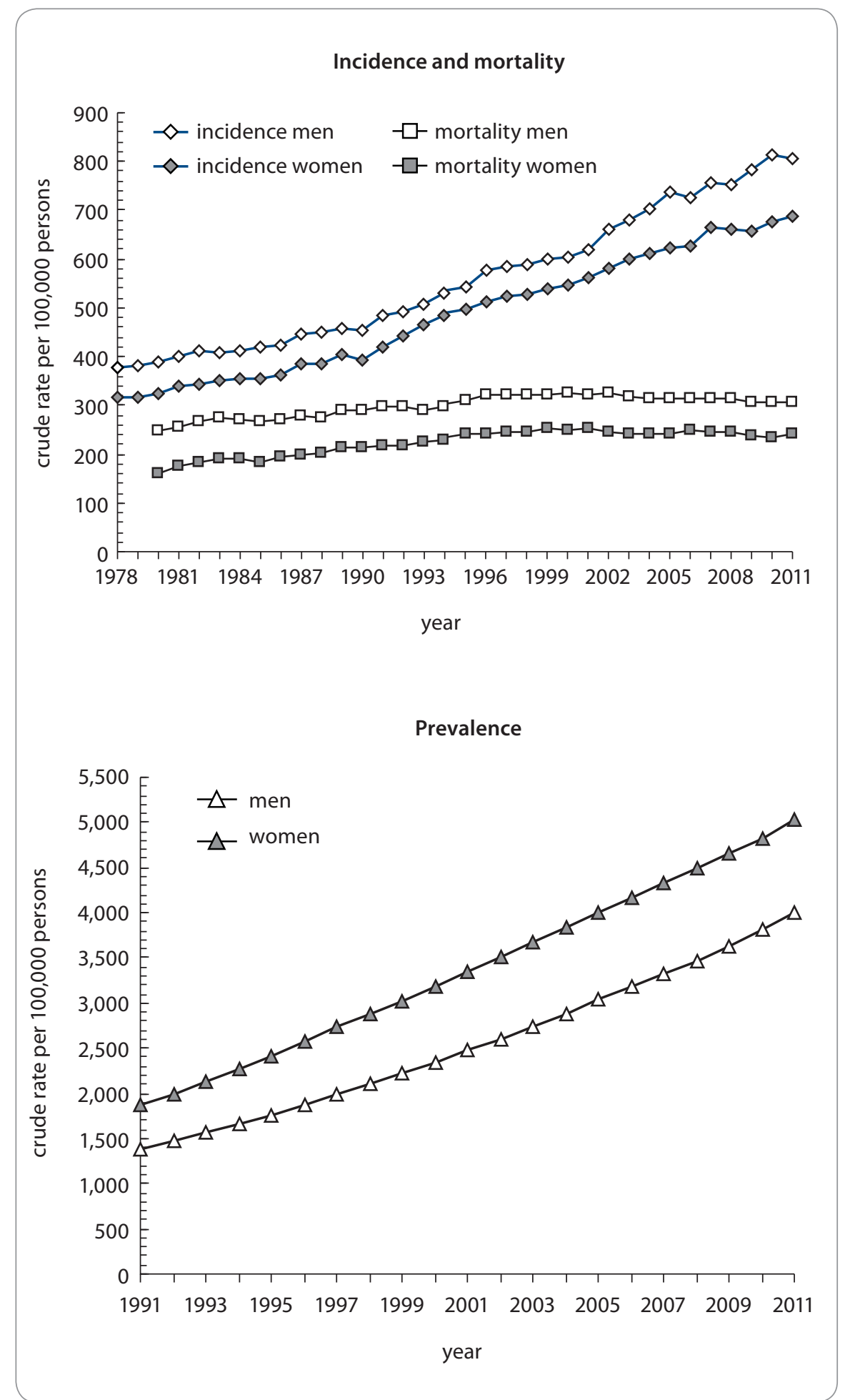

Fig. 1. Time trends of cancer incidence, mortality, and prevalence in the Czech Republic (all diagnoses including non-melanoma skin cancer, C00-C97).

calculations take into consideration the epidemiological situation in a given region, from which weights are derived to distribute the population-based prediction. is illustrated in Tab. 1. In 2011, 77,773 new incident cases (740.3 per 100,000 individuals) of all cancers including skin neoplasms (COO-C97) were diagnosed. The crude incidence rate continuously increases with the growth index $+27.6 \%$ in the last decade (2001-2011). Growing incidence and stabilized mortality necessarily increase prevalence which exceeded 475,000 of cases in 2011. Time trends in incidence, mortality and prevalence (calculated per 100,000 people) of all malignant tumors including skin neoplasms (C00-C97) in the Czech Republic according to patient's sex are given in Fig. 1.

The predicted values of stage-specific incidence for years 2015 and 2020 are shown in Tab. 2 and 3, respectively. We can see that 9,693 newly diagnosed prostate cancer cases are expected in 2015 in the Czech Republic (corresponding to $39 \%$ increase with respect to 2011 [3]) introducing prostate cancer as the most incident diagnosis of all malignant neoplasms in 2015. This trend is also expected in 2020 with estimated 13,153 newly diagnosed prostate cancer cases $(89 \%$ increase with respect to 2011), followed by 9,368 (15\% increase with respect to 2011), 8,695 (31\% increase with respect to 2011 ), and 8,604 (32\% increase with respect to 2011) newly diagnosed colorectal, female breast, and lung cancer cases, respectively.

Tables 4 and 5 show the predicted values of stage-specific one-year prevalence for all considered cancer diagnoses in 2015 and 2020, respectively. According to the model, we can expect more than 85,000 prevalent females with history of breast cancer $(21 \%$ increase with respect to 2011 [3]), almost 66,000 colorectal cancer patients (16\% increase with respect to 2011), and more than 65,000 prostate cancer patients $(52 \%$ increase with respect to 2011) in the Czech Republic in 2015. In lung cancer, only $6 \%$ increase in prevalence (to 18,401 patients in total) is expected in 2015 with respect to 2011. In 2020, more than 100,000 prevalent femalebreastcancer patients areexpected in the Czech Republic (51\% increase 
Tab. 4. Predicted values of cancer prevalence in the Czech Republic in 2015 according to diagnosis and clinical stage of primary tumor. Numbers of patients are accompanied with $90 \%$ confidence intervals (in brackets).

\begin{tabular}{|c|c|c|c|c|c|c|}
\hline Diagnosis (ICD-10 code) & Stage I & Stage II & Stage III & Stage IV & $\begin{array}{c}\text { Stage } \\
\text { unknown }\end{array}$ & Total \\
\hline $\begin{array}{l}\text { head and neck } \\
\text { (C00-C14, C30-C31) }\end{array}$ & $\begin{array}{c}2,382 \\
(2,302 ; 2,462)\end{array}$ & $\begin{array}{c}1,316 \\
(1,256 ; 1,376)\end{array}$ & $\begin{array}{c}1,558 \\
(1,493 ; 1,623)\end{array}$ & $\begin{array}{c}3,600 \\
(3,501 ; 3,699)\end{array}$ & $\begin{array}{c}1,024 \\
(971 ; 1,077)\end{array}$ & $\begin{array}{c}9,880 \\
(9,717 ; 10,043)\end{array}$ \\
\hline $\begin{array}{l}\text { oesophagus } \\
\text { (C15) }\end{array}$ & $\begin{array}{c}162 \\
(141 ; 183)\end{array}$ & $\begin{array}{c}502 \\
(465 ; 539)\end{array}$ & $\begin{array}{c}401 \\
(368 ; 434)\end{array}$ & $\begin{array}{c}663 \\
(621 ; 705)\end{array}$ & $\begin{array}{c}93 \\
(77 ; 109)\end{array}$ & $\begin{array}{c}1,821 \\
(1,751 ; 1,891)\end{array}$ \\
\hline $\begin{array}{l}\text { stomach } \\
\text { (C16) }\end{array}$ & $\begin{array}{c}2,589 \\
(2,505 ; 2,673)\end{array}$ & $\begin{array}{c}1,137 \\
(1,082 ; 1,192)\end{array}$ & $\begin{array}{c}880 \\
(831 ; 929)\end{array}$ & $\begin{array}{c}1,966 \\
(1,893 ; 2,039)\end{array}$ & $\begin{array}{c}718 \\
(674 ; 762)\end{array}$ & $\begin{array}{c}7,290 \\
(7,150 ; 7,430)\end{array}$ \\
\hline $\begin{array}{l}\text { colorectal cancer } \\
\text { (C18-C20) }\end{array}$ & $\begin{array}{c}21,303 \\
(21,063 ; 21,543)\end{array}$ & $\begin{array}{c}19,019 \\
(18,792 ; 19,246)\end{array}$ & $\begin{array}{c}14,701 \\
(14,502 ; 14,900)\end{array}$ & $\begin{array}{c}8,388 \\
(8,237 ; 8,539)\end{array}$ & $\begin{array}{c}2,399 \\
(2,318 ; 2,480)\end{array}$ & $\begin{array}{c}65,810 \\
(65,388 ; 66,232)\end{array}$ \\
\hline $\begin{array}{l}\text { pancreas } \\
\text { (C25) }\end{array}$ & \multicolumn{2}{|c|}{$\begin{array}{c}1,219 \\
(1,162 ; 1,276)\end{array}$} & $\begin{array}{c}316 \\
(287 ; 345)\end{array}$ & $\begin{array}{c}2,307 \\
(2,228 ; 2,386)\end{array}$ & $\begin{array}{c}464 \\
(429 ; 499)\end{array}$ & $\begin{array}{c}4,306 \\
(4,198 ; 4,414)\end{array}$ \\
\hline $\begin{array}{l}\operatorname{larynx} \\
\text { (C32) }\end{array}$ & $\begin{array}{c}1,914 \\
(1,842 ; 1,986)\end{array}$ & $\begin{array}{c}945 \\
(894 ; 996)\end{array}$ & $\begin{array}{c}885 \\
(836 ; 934)\end{array}$ & $\begin{array}{c}1,049 \\
(996 ; 1,102)\end{array}$ & $\begin{array}{c}128 \\
(109 ; 147)\end{array}$ & $\begin{array}{c}4,921 \\
(4,806 ; 5,036)\end{array}$ \\
\hline $\begin{array}{l}\text { lung } \\
\text { (C34) }\end{array}$ & $\begin{array}{c}3,962 \\
(3,858 ; 4,066)\end{array}$ & $\begin{array}{c}1,260 \\
(1,202 ; 1,318)\end{array}$ & $\begin{array}{c}3,517 \\
(3,419 ; 3,615)\end{array}$ & $\begin{array}{c}8,413 \\
(8,262 ; 8,564)\end{array}$ & $\begin{array}{c}1,249 \\
(1,191 ; 1,307)\end{array}$ & $\begin{array}{c}18,401 \\
(18,178 ; 18,624)\end{array}$ \\
\hline $\begin{array}{l}\text { melanoma of skin } \\
\text { (C43) }\end{array}$ & $\begin{array}{c}21,346 \\
(21,106 ; 21,586)\end{array}$ & $\begin{array}{c}3,771 \\
(3,670 ; 3,872)\end{array}$ & $\begin{array}{c}1,167 \\
(1,111 ; 1,223)\end{array}$ & $\begin{array}{c}744 \\
(699 ; 789)\end{array}$ & $\begin{array}{c}794 \\
(748 ; 840)\end{array}$ & $\begin{array}{c}27,822 \\
(27,548 ; 28,096)\end{array}$ \\
\hline $\begin{array}{l}\text { female breast } \\
\text { (C50) }\end{array}$ & $\begin{array}{c}38,041 \\
(37,720 ; 38,362)\end{array}$ & $\begin{array}{c}32,783 \\
(32,485 ; 33,081)\end{array}$ & $\begin{array}{c}9,454 \\
(9,294 ; 9,614)\end{array}$ & $\begin{array}{c}3,411 \\
(3,315 ; 3,507)\end{array}$ & $\begin{array}{c}1,689 \\
(1,621 ; 1,757)\end{array}$ & $\begin{array}{c}85,378 \\
(84,897 ; 85,859)\end{array}$ \\
\hline $\begin{array}{l}\text { cervix uteri } \\
\text { (C53) }\end{array}$ & $\begin{array}{c}12,675 \\
(12,490 ; 12,860)\end{array}$ & $\begin{array}{c}2,344 \\
(2,264 ; 2,424)\end{array}$ & $\begin{array}{c}2,007 \\
(1,933 ; 2,081)\end{array}$ & $\begin{array}{c}614 \\
(573 ; 655)\end{array}$ & $\begin{array}{c}1,176 \\
(1,120 ; 1,232)\end{array}$ & $\begin{array}{c}18,816 \\
(18,590 ; 19,042)\end{array}$ \\
\hline $\begin{array}{l}\text { uterus } \\
\text { (C54) }\end{array}$ & $\begin{array}{c}22,559 \\
(22,312 ; 22,806)\end{array}$ & $\begin{array}{c}2,483 \\
(2,401 ; 2,565)\end{array}$ & $\begin{array}{c}1,719 \\
(1,651 ; 1,787)\end{array}$ & $\begin{array}{c}665 \\
(623 ; 707)\end{array}$ & $\begin{array}{c}2,801 \\
(2,714 ; 2,888)\end{array}$ & $\begin{array}{c}30,227 \\
(29,941 ; 30,513)\end{array}$ \\
\hline $\begin{array}{l}\text { ovary } \\
\text { (C56) }\end{array}$ & $\begin{array}{c}4,760 \\
(4,647 ; 4,873)\end{array}$ & $\begin{array}{c}1,005 \\
(953 ; 1,057)\end{array}$ & $\begin{array}{c}2,640 \\
(2,555 ; 2,725)\end{array}$ & $\begin{array}{c}1,592 \\
(1,526 ; 1,658)\end{array}$ & $\begin{array}{c}644 \\
(602 ; 686)\end{array}$ & $\begin{array}{c}10,641 \\
(10,471 ; 10,811)\end{array}$ \\
\hline $\begin{array}{l}\text { prostate } \\
\text { (C61) }\end{array}$ & \multicolumn{2}{|c|}{$\begin{array}{c}47,454 \\
(47,096 ; 47,812)\end{array}$} & $\begin{array}{c}8,492 \\
(8,340 ; 8,644)\end{array}$ & $\begin{array}{c}5,884 \\
(5,758 ; 6,010)\end{array}$ & $\begin{array}{c}3,384 \\
(3,288 ; 3,480)\end{array}$ & $\begin{array}{c}65,214 \\
(64,794 ; 65,634)\end{array}$ \\
\hline $\begin{array}{l}\text { testis } \\
\text { (C62) }\end{array}$ & $\begin{array}{c}6,260 \\
(6,130 ; 6,390)\end{array}$ & $\begin{array}{c}1,743 \\
(1,674 ; 1,812)\end{array}$ & $\begin{array}{c}842 \\
(794 ; 890)\end{array}$ & * & $\begin{array}{c}837 \\
(789 ; 885)\end{array}$ & $\begin{array}{c}9,682 \\
(9,520 ; 9,844)\end{array}$ \\
\hline $\begin{array}{l}\text { kidney } \\
\text { (C64) }\end{array}$ & \multicolumn{2}{|c|}{$\begin{array}{c}18,772 \\
(18,547 ; 18,997)\end{array}$} & $\begin{array}{c}2,919 \\
(2,830 ; 3,008)\end{array}$ & $\begin{array}{c}2,360 \\
(2,280 ; 2,440)\end{array}$ & $\begin{array}{c}1,779 \\
(1,710 ; 1,848)\end{array}$ & $\begin{array}{c}25,830 \\
(25,566 ; 26,094)\end{array}$ \\
\hline $\begin{array}{l}\text { bladder } \\
\text { (C67) }\end{array}$ & $\begin{array}{c}14,505 \\
(14,307 ; 14,703)\end{array}$ & $\begin{array}{c}3,164 \\
(3,071 ; 3,257)\end{array}$ & $\begin{array}{c}640 \\
(598 ; 682)\end{array}$ & $\begin{array}{c}1,382 \\
(1,321 ; 1,443)\end{array}$ & $\begin{array}{c}2,147 \\
(2,071 ; 2,223)\end{array}$ & $\begin{array}{c}21,838 \\
(21,595 ; 22,081)\end{array}$ \\
\hline $\begin{array}{l}\text { brain } \\
\text { (C71) }\end{array}$ & $* *$ & $* *$ & ** & ** & ** & $\begin{array}{c}3,763 \\
(3,662 ; 3,864)\end{array}$ \\
\hline $\begin{array}{l}\text { thyroid } \\
\text { (C73) }\end{array}$ & $\begin{array}{c}8,880 \\
(8,725 ; 9,035)\end{array}$ & $\begin{array}{c}2,210 \\
(2,133 ; 2,287)\end{array}$ & $\begin{array}{c}1,226 \\
(1,168 ; 1,284)\end{array}$ & $\begin{array}{c}693 \\
(650 ; 736)\end{array}$ & $\begin{array}{c}1,307 \\
(1,248 ; 1,366)\end{array}$ & $\begin{array}{c}14,316 \\
(14,119 ; 14,513)\end{array}$ \\
\hline $\begin{array}{l}\text { Hodgkin lymphoma } \\
\text { (C81) }\end{array}$ & $\begin{array}{c}927 \\
(877 ; 977)\end{array}$ & $\begin{array}{c}2,255 \\
(2,177 ; 2,333)\end{array}$ & $\begin{array}{c}1,147 \\
(1,091 ; 1,203)\end{array}$ & $\begin{array}{c}519 \\
(482 ; 556)\end{array}$ & $\begin{array}{c}957 \\
(906 ; 1,008)\end{array}$ & $\begin{array}{c}5,805 \\
(5,680 ; 5,930)\end{array}$ \\
\hline $\begin{array}{l}\text { non-Hodgkin lymphoma } \\
\text { (C82-C85) }\end{array}$ & $\begin{array}{c}2,406 \\
(2,325 ; 2,487)\end{array}$ & $\begin{array}{c}2,589 \\
(2,505 ; 2,673)\end{array}$ & $\begin{array}{c}1,975 \\
(1,902 ; 2,048)\end{array}$ & $\begin{array}{c}2,365 \\
(2,285 ; 2,445)\end{array}$ & $\begin{array}{c}3,046 \\
(2,955 ; 3,137)\end{array}$ & $\begin{array}{c}12,381 \\
(12,198 ; 12,564)\end{array}$ \\
\hline $\begin{array}{l}\text { multiple myeloma } \\
\text { (C90) }\end{array}$ & ** & ** & ** & ** & ** & $\begin{array}{c}2,797 \\
(2,710 ; 2,884)\end{array}$ \\
\hline
\end{tabular}

* Stage IV is not defined for testicular cancer (C62).

**Clinical stage was not considered in models for brain tumors (C71) and multiple myeloma (C90). 
Tab. 5. Predicted values of cancer prevalence in the Czech Republic in 2020 according to diagnosis and clinical stage of primary tumor. Numbers of patients are accompanied with $90 \%$ confidence intervals (in brackets).

\begin{tabular}{|c|c|c|c|c|c|c|}
\hline Diagnosis (ICD-10 code) & Stage I & Stage II & Stage III & Stage IV & $\begin{array}{c}\text { Stage } \\
\text { unknown }\end{array}$ & Total \\
\hline $\begin{array}{l}\text { head and neck } \\
\text { (C00-C14, C30-C31) }\end{array}$ & $\begin{array}{c}2,580 \\
(2,496 ; 2,664)\end{array}$ & $\begin{array}{c}1,429 \\
(1,367 ; 1,491)\end{array}$ & $\begin{array}{c}1,774 \\
(1,705 ; 1,843)\end{array}$ & $\begin{array}{c}4,287 \\
(4,179 ; 4,395)\end{array}$ & $\begin{array}{c}1,069 \\
(1,015 ; 1,123)\end{array}$ & $\begin{array}{c}11,139 \\
(10,965 ; 11,313)\end{array}$ \\
\hline $\begin{array}{l}\text { oesophagus } \\
\text { (C15) }\end{array}$ & $\begin{array}{c}213 \\
(189 ; 237)\end{array}$ & $\begin{array}{c}669 \\
(626 ; 712)\end{array}$ & $\begin{array}{c}518 \\
(481 ; 555)\end{array}$ & $\begin{array}{c}859 \\
(811 ; 907)\end{array}$ & $\begin{array}{c}49 \\
(37 ; 61)\end{array}$ & $\begin{array}{c}2,308 \\
(2,229 ; 2,387)\end{array}$ \\
\hline $\begin{array}{l}\text { stomach } \\
\text { (C16) }\end{array}$ & $\begin{array}{c}3,059 \\
(2,968 ; 3,150)\end{array}$ & $\begin{array}{c}1,238 \\
(1,180 ; 1,296)\end{array}$ & $\begin{array}{c}996 \\
(944 ; 1,048)\end{array}$ & $\begin{array}{c}2,451 \\
(2,370 ; 2,532)\end{array}$ & $\begin{array}{c}765 \\
(720 ; 810)\end{array}$ & $\begin{array}{c}8,509 \\
(8,357 ; 8,661)\end{array}$ \\
\hline $\begin{array}{l}\text { colorectal cancer } \\
\text { (C18-C20) }\end{array}$ & $\begin{array}{c}26,992 \\
(26,722 ; 27,262)\end{array}$ & $\begin{array}{c}21,551 \\
(21,310 ; 21,792)\end{array}$ & $\begin{array}{c}19,062 \\
(18,835 ; 19,289)\end{array}$ & $\begin{array}{c}9,946 \\
(9,782 ; 10,110)\end{array}$ & $\begin{array}{c}2,035 \\
(1,961 ; 2,109)\end{array}$ & $\begin{array}{c}79,586 \\
(79,122 ; 80,050)\end{array}$ \\
\hline $\begin{array}{l}\text { pancreas } \\
\text { (C25) }\end{array}$ & \multicolumn{2}{|c|}{$\begin{array}{c}1,702 \\
(1,634 ; 1,770)\end{array}$} & $\begin{array}{c}375 \\
(343 ; 407)\end{array}$ & $\begin{array}{c}2,748 \\
(2,662 ; 2,834)\end{array}$ & $\begin{array}{c}355 \\
(324 ; 386)\end{array}$ & $\begin{array}{c}5,180 \\
(5,062 ; 5,298)\end{array}$ \\
\hline $\begin{array}{l}\operatorname{larynx} \\
\text { (C32) }\end{array}$ & $\begin{array}{c}2,049 \\
(1,975 ; 2,123)\end{array}$ & $\begin{array}{c}1,093 \\
(1,039 ; 1,147)\end{array}$ & $\begin{array}{c}975 \\
(924 ; 1,026)\end{array}$ & $\begin{array}{c}1,181 \\
(1,124 ; 1,238)\end{array}$ & $\begin{array}{c}89 \\
(73 ; 105)\end{array}$ & $\begin{array}{c}5,387 \\
(5,266 ; 5,508)\end{array}$ \\
\hline $\begin{array}{l}\text { lung } \\
\text { (C34) }\end{array}$ & $\begin{array}{c}4,392 \\
(4,283 ; 4,501)\end{array}$ & $\begin{array}{c}1,154 \\
(1,098 ; 1,210)\end{array}$ & $\begin{array}{c}3,673 \\
(3,573 ; 3,773)\end{array}$ & $\begin{array}{c}10,127 \\
(9,961 ; 10,293)\end{array}$ & $\begin{array}{c}906 \\
(856 ; 956)\end{array}$ & $\begin{array}{c}20,252 \\
(20,018 ; 20,486)\end{array}$ \\
\hline $\begin{array}{l}\text { melanoma of skin } \\
\text { (C43) }\end{array}$ & $\begin{array}{c}28,764 \\
(28,485 ; 29,043)\end{array}$ & $\begin{array}{c}4,475 \\
(4,365 ; 4,585)\end{array}$ & $\begin{array}{c}1,240 \\
(1,182 ; 1,298)\end{array}$ & $\begin{array}{c}845 \\
(797 ; 893)\end{array}$ & $\begin{array}{c}773 \\
(727 ; 819)\end{array}$ & $\begin{array}{c}36,097 \\
(35,784 ; 36,410)\end{array}$ \\
\hline $\begin{array}{l}\text { female breast } \\
\text { (C50) }\end{array}$ & $\begin{array}{c}52,269 \\
(51,893 ; 52,645)\end{array}$ & $\begin{array}{c}37,555 \\
(37,236 ; 37,874)\end{array}$ & $\begin{array}{c}12,450 \\
(12,266 ; 12,634)\end{array}$ & $\begin{array}{c}3,519 \\
(3,421 ; 3,617)\end{array}$ & $\begin{array}{c}1,175 \\
(1,119 ; 1,231)\end{array}$ & $\begin{array}{c}106,968 \\
(106,430 ; 107,506)\end{array}$ \\
\hline $\begin{array}{l}\text { cervix uteri } \\
\text { (C53) }\end{array}$ & $\begin{array}{c}13,841 \\
(13,647 ; 14,035)\end{array}$ & $\begin{array}{c}2,236 \\
(2,158 ; 2,314)\end{array}$ & $\begin{array}{c}2,153 \\
(2,077 ; 2,229)\end{array}$ & $\begin{array}{c}692 \\
(649 ; 735)\end{array}$ & $\begin{array}{c}1,062 \\
(1,008 ; 1,116)\end{array}$ & $\begin{array}{c}19,984 \\
(19,751 ; 20,217)\end{array}$ \\
\hline $\begin{array}{l}\text { uterus } \\
\text { (C54) }\end{array}$ & $\begin{array}{c}27,163 \\
(26,892 ; 27,434)\end{array}$ & $\begin{array}{c}3,193 \\
(3,100 ; 3,286)\end{array}$ & $\begin{array}{c}2,340 \\
(2,260 ; 2,420)\end{array}$ & $\begin{array}{c}707 \\
(663 ; 751)\end{array}$ & $\begin{array}{c}3,074 \\
(2,983 ; 3,165)\end{array}$ & $\begin{array}{c}36,477 \\
(36,163 ; 36,791)\end{array}$ \\
\hline $\begin{array}{l}\text { ovary } \\
\text { (C56) }\end{array}$ & $\begin{array}{c}5,289 \\
(5,169 ; 5,409)\end{array}$ & $\begin{array}{c}1,131 \\
(1,076 ; 1,186)\end{array}$ & $\begin{array}{c}3,193 \\
(3,100 ; 3,286)\end{array}$ & $\begin{array}{c}1,649 \\
(1,582 ; 1,716)\end{array}$ & $\begin{array}{c}516 \\
(479 ; 553)\end{array}$ & $\begin{array}{c}11,778 \\
(11,599 ; 11,957)\end{array}$ \\
\hline $\begin{array}{l}\text { prostate } \\
\text { (C61) }\end{array}$ & \multicolumn{2}{|c|}{$\begin{array}{c}79,230 \\
(78,767 ; 79693)\end{array}$} & $\begin{array}{c}13,930 \\
(13,736 ; 14,124)\end{array}$ & $\begin{array}{c}7,448 \\
(7,306 ; 7,590)\end{array}$ & $\begin{array}{c}2,374 \\
(2,294 ; 2,454)\end{array}$ & $\begin{array}{c}102,982 \\
(102,454 ; 103,510)\end{array}$ \\
\hline $\begin{array}{l}\text { testis } \\
\text { (C62) }\end{array}$ & $\begin{array}{c}7,751 \\
(7,606 ; 7,896)\end{array}$ & $\begin{array}{c}1,972 \\
(1,899 ; 2,045)\end{array}$ & $\begin{array}{c}906 \\
(856 ; 956)\end{array}$ & * & $\begin{array}{c}803 \\
(756 ; 850)\end{array}$ & $\begin{array}{c}11,432 \\
(11,256 ; 11,608)\end{array}$ \\
\hline $\begin{array}{l}\text { kidney } \\
\text { (C64) }\end{array}$ & \multicolumn{2}{|c|}{$\begin{array}{c}22,966 \\
(22,717 ; 23,215)\end{array}$} & $\begin{array}{c}3,176 \\
(3,083 ; 3,269)\end{array}$ & $\begin{array}{c}2,665 \\
(2,580 ; 2,750)\end{array}$ & $\begin{array}{c}1,487 \\
(1,424 ; 1,550)\end{array}$ & $\begin{array}{c}30,294 \\
(30,008 ; 30,580)\end{array}$ \\
\hline $\begin{array}{l}\text { bladder } \\
\text { (C67) }\end{array}$ & $\begin{array}{c}17,795 \\
(17,576 ; 18,014)\end{array}$ & $\begin{array}{c}3,494 \\
(3,397 ; 3,591)\end{array}$ & $\begin{array}{c}676 \\
(633 ; 719)\end{array}$ & $\begin{array}{c}1,705 \\
(1,637 ; 1,773)\end{array}$ & $\begin{array}{c}1,397 \\
(1,336 ; 1,458)\end{array}$ & $\begin{array}{c}25,067 \\
(24,807 ; 25,327)\end{array}$ \\
\hline $\begin{array}{l}\text { brain } \\
\text { (C71) }\end{array}$ & $* *$ & $* *$ & $* *$ & $* *$ & $* *$ & $\begin{array}{c}4,207 \\
(4,100 ; 4,314)\end{array}$ \\
\hline $\begin{array}{l}\text { thyroid } \\
\text { (C73) }\end{array}$ & $\begin{array}{c}13,350 \\
(13,160 ; 13,540)\end{array}$ & $\begin{array}{c}2,113 \\
(2,037 ; 2,189)\end{array}$ & $\begin{array}{c}1,520 \\
(1,456 ; 1,584)\end{array}$ & $\begin{array}{c}889 \\
(840 ; 938)\end{array}$ & $\begin{array}{c}1,624 \\
(1,558 ; 1,690)\end{array}$ & $\begin{array}{c}19,496 \\
(19,266 ; 19,726)\end{array}$ \\
\hline $\begin{array}{l}\text { Hodgkin lymphoma } \\
\text { (C81) }\end{array}$ & $\begin{array}{c}1,006 \\
(954 ; 1,058)\end{array}$ & $\begin{array}{c}2,637 \\
(2,553 ; 2,721)\end{array}$ & $\begin{array}{c}1,364 \\
(1,303 ; 1,425)\end{array}$ & $\begin{array}{c}565 \\
(526 ; 604)\end{array}$ & $\begin{array}{c}888 \\
(839 ; 937)\end{array}$ & $\begin{array}{c}6,460 \\
(6,328 ; 6,592)\end{array}$ \\
\hline $\begin{array}{l}\text { non-Hodgkin lymphoma } \\
\text { (C82-C85) }\end{array}$ & $\begin{array}{c}3,036 \\
(2,945 ; 3,127)\end{array}$ & $\begin{array}{c}4,026 \\
(3,922 ; 4,130)\end{array}$ & $\begin{array}{c}3,089 \\
(2,998 ; 3,180)\end{array}$ & $\begin{array}{c}3,141 \\
(3,049 ; 3,233)\end{array}$ & $\begin{array}{c}3,105 \\
(3,013 ; 3,197)\end{array}$ & $\begin{array}{c}16,397 \\
(16,186 ; 16,608)\end{array}$ \\
\hline $\begin{array}{l}\text { multiple myeloma } \\
\text { (C90) }\end{array}$ & $* *$ & $* *$ & $* *$ & ** & $* *$ & $\begin{array}{c}3,409 \\
(3,313 ; 3,505)\end{array}$ \\
\hline
\end{tabular}

* Stage IV is not defined for testicular cancer (C62).

**Clinical stage was not considered in models for brain tumors (C71) and multiple myeloma (C90). 
Tab. 6. Stage-specific estimates of prevalence of patients requiring active anti-tumor therapy for colorectal cancer in the Czech Republic in 2015 and 2020. Numbers of patients are accompanied with 90\% confidence intervals (in brackets).

Colorectal cancer (C18-C20)

Newly diagnosed cancer patients

\section{year 2015}

stage I

stage II

stage III

stage IV

stage unknown

number of treated patients in given category

total number of treated patients

\section{Non-terminal recurrence in patients from previous years}

\section{Terminal recurrence in patients from previous years}

\author{
year 2020 \\ stage 1 \\ stage 2 \\ stage 3 \\ stage 4 \\ stage unknown \\ number of treated patients in given \\ category
}

total number of treated patients

$1,890(1,744 ; 2,035)$
$1,829(1,726 ; 1,933)$
$1,915(1,776 ; 2,054)$
$1,473(1,398 ; 1,550)$
$57(40 ; 75)$

7,164

$(6,684 ; 7,647)$
$793(747 ; 839)$

$1,022(969 ; 1,075)$

$1,247(1,189 ; 1,305)$

$679(636 ; 722)$

$21(13 ; 29)$

3,762

$(3,661 ; 3,863)$

$(1,678 ; 1,816)$
$12,673(12,488 ; 12,858)$

*Patients with terminal cancer recurrence are assumed to be treated with generalised disease.

with respect to 2011), as well as more than 100,000 prevalent prostate cancer patients $(140 \%$ increase with respect to 2011). In colorectal and lung cancer, $40 \%$ and $17 \%$ increase in prevalence is expected in 2020 with respect to 2011.

The numbers of patients requiring active anti-tumor therapy for colorectal, lung, female breast and prostate cancer in the Czech Republic in 2015 and 2020 are given in Tab. 6-9. For each cancer diagnosis, the three columns represent the individual components of the proposed model - the estimated number of newly diagnosed and treated patients, the estimated number of patients treated for non-terminal cancer recurrence, and the estimated number of patients treated for terminal cancer recurrence. For example, we can see that 12,673 and 14,006 colorectal cancers are expected to be treated in the Czech Republic in 2015 and 2020, respectively (Tab. 6). Regarding the overall number of these patients treated in stage IV, 3,899 (31\%) and $4,190(30 \%)$ colorectal cancer patients are predicted to be treated for the metastatic disease in 2015 and 2020, respectively. Similar estimates can be observed for female breast cancer 12,827 and 14,759 females requiring active anti-tumor therapy for breast cancer in 2015 and 2020, respectively (Tab. 8). However, only $15 \%(1,932)$ and $13 \%(1,958)$ are expected to be treated for the metastatic disease in 2015 and 2020, respectively.

The numbers of patients requiring active anti-tumor therapy were also localized to individual regions of the Czech Republic. An example of such analysis for colorectal cancer is shown in Fig. 2.

\section{Discussion}

Czech Republic is among countries with the highest cancer burden where mortality from cancer contributes $25.8 \%$ to the overall population mortality. Moreover, we can expect the overall cancer burden to grow even in the future $[4,5]$. The current role of cancer epidemiology is not purely descriptive. Knowledge of age-, stage-, and region-specific trends is necessary to evaluate the effectiveness of diagnostic processes, to identify weak points in the management of cancer care and to analyze therapeutic outcomes. Moreover, to be able to monitor and evaluate the cancer patient population dynamics, it is necessary to prospectively model incidence and prevalence rates. In this paper, we present the stage-specific predictions of cancer incidence and prevalence, and 
Tab. 7. Stage-specific estimates of prevalence of patients requiring active anti-tumor therapy for lung cancer in the Czech Republic in 2015 and 2020. Numbers of patients are accompanied with $90 \%$ confidence intervals (in brackets).

\begin{tabular}{|c|c|c|c|}
\hline Lung (C34) & $\begin{array}{l}\text { Newly diagnosed cancer } \\
\text { patients }\end{array}$ & $\begin{array}{c}\text { Non-terminal recurrence } \\
\text { in patients from } \\
\text { previous years }\end{array}$ & $\begin{array}{l}\text { Terminal recurrence } \\
\text { in patients from } \\
\text { previous years }\end{array}$ \\
\hline \multicolumn{4}{|l|}{ year 2015} \\
\hline stage I & $594(541 ; 647)$ & $199(176 ; 222)$ & \\
\hline stage II & $237(207 ; 266)$ & $82(67 ; 97)$ & \\
\hline stage III & $1,229(1,093 ; 1,364)$ & $319(290 ; 348)$ & \\
\hline stage IV & $2,449(2,308 ; 2,589)$ & $337(307 ; 367)$ & $1,594(1,528 ; 1,660)^{*}$ \\
\hline stage unknown & $127(79 ; 176)$ & $9(4 ; 14)$ & \\
\hline $\begin{array}{l}\text { number of treated patients in given } \\
\text { category }\end{array}$ & $\begin{array}{c}4,636 \\
(4,228 ; 5,042)\end{array}$ & $\begin{array}{c}946 \\
(895 ; 997)\end{array}$ & $\begin{array}{c}1,594 \\
(1,528 ; 1,660)\end{array}$ \\
\hline total number of treated patients & & $7,176(7,037 ; 7,315)$ & \\
\hline \multicolumn{4}{|l|}{ year 2020} \\
\hline stage I & $699(627 ; 772)$ & $229(204 ; 254)$ & \\
\hline stage II & $229(193 ; 265)$ & $71(57 ; 85)$ & \\
\hline stage III & $1,331(1,135 ; 1,526)$ & $337(307 ; 367)$ & \\
\hline stage IV & $3,037(2,841 ; 3,231)$ & $416(382 ; 450)$ & $1,829(1,759 ; 1,899)^{*}$ \\
\hline stage unknown & $88(44 ; 131)$ & $6(2 ; 10)$ & \\
\hline $\begin{array}{l}\text { number of treated patients in given } \\
\text { category }\end{array}$ & $\begin{array}{c}5,384 \\
(4,840 ; 5,925)\end{array}$ & $\begin{array}{c}1,059 \\
(1,005 ; 1,113)\end{array}$ & $\begin{array}{c}1,829 \\
(1,759 ; 1,899)\end{array}$ \\
\hline total number of treated patients & & $8,272(8,122 ; 8,422)$ & \\
\hline
\end{tabular}

the stage- and region-specific estimates of patients requiring active anti-tumor therapy in the Czech Republic for years 2015 and 2020.

The analysis, which utilizes solely the population-based cancer registry data, documents a serious increase in cancer incidence and prevalence in the Czech Republic in years 2015 and 2020 when compared to the situation in 2011. For example, enormous increase in prostate cancer incidence and prevalence is expected in 2020 with respect to $2011: 89 \%$ and $140 \%$, respectively. Such findings are concordant with results published for other European states $[1,18]$. However, the incidence of prostate cancer will depend on the existence of prostate cancer screening programs. The benefit of screening programs has not been firmly established and it is therefore impossible to predict whether they will be implemented. In the US, we are currently witnessing a decrease of prostate cancer incidence after a peak caused by the onset of prostate cancer screening in the 1980s. On the other hand, in lung cancer, we expect $32 \%$ increase in incidence in 2020 but only $17 \%$ increase in prevalence in 2020 with respect to 2011 . The reason for such a discrepancy is simple different stage distribution and the associated stage-specific mortality of both diagnoses. Not only epidemiology characteristics but also the number of cancer patients requiring active anti-tumor therapy is expected to grow steadily in the Czech Republic up to year 2020. We expect an increase of $11 \%, 15 \%, 15 \%$, and $42 \%$ in the number of patients probably treated for colorectal, lung, female breast, and prostate cancer, respectively, in the Czech Republic between years 2015 and 2020. Increasing numbers of cancer patients treated in the continuing care phase resulting from the increase in the number of cancer survivors were reported also in the US study [19].

The estimation of the stage-specific prevalence is a challenging task with a relatively little information on this subject in the literature [9]. Disregarding the stage-specific nature, several methods have been proposed for future cancer burden estimation based on different modelling strategies. The back-calculation method, combining parametric estimates of incidence and survival, is the most frequently used. The PIAMOD model [20] has been repeatedly used for projections of cancer prevalence utilizing the age-period-cohort model for cancer 
Tab. 8. Stage-specific estimates of prevalence of patients requiring active anti-tumor therapy for breast cancer (females) in the Czech Republic in 2015 and 2020. Numbers of patients are accompanied with 90\% confidence intervals (in brackets).

Female breast (C50)

\section{year 2015}

stage I

stage II

stage III

stage IV

stage unknown

number of treated patients in given

category

total number of treated patients

\section{Newly diagnosed cancer Non-terminal recurrence patients \\ in patients from \\ previous years}

\section{Terminal recurrence in patients from previous years}

\section{year 2020}

stage I

$3,402(3,071 ; 3,731)$

$2,213(2,022 ; 2,406)$

$1,131(1,009 ; 1,254)$

$411(352 ; 472)$

$28(15 ; 42)$

7,185

$(6,469 ; 7,905)$
$1,590(1,524 ; 1,656)$

$1,621(1,555 ; 1,687)$

$891(842 ; 940)$

$286(258 ; 314)$

$19(12 ; 26)$

4,407

$(4,298 ; 4,516)$

$(1,177 ; 1,293)$
$1,235(1,177 ; 1,293)^{*}$

$12,827(12,641 ; 13,013)$

stage II

stage III

stage IV

stage unknown

number of treated patients in given category

total number of treated patients

$4,170(3,720 ; 4,619)$
$2,338(2,094 ; 2,583)$
$1,405(1,234 ; 1,577)$
$442(360 ; 525)$
$14(5 ; 24)$

8,369

$(7,413 ; 9,328)$
$2,005(1,931 ; 2,079)$

$1,701(1,633 ; 1,769)$

$1,158(1,102 ; 1,214)$ $309(280 ; 338)$

$10(5 ; 15)$

5,183

$(5,065 ; 5,301)$

1,207

$(1,150 ; 1,264)$

*Patients with terminal cancer recurrence are assumed to be treated with generalised disease.

incidence and age-drift model for extrapolations. Cure fraction model is employed for relative survival estimation, including period effect useful for extrapolation of survival into future time periods. In principle, our model uses similar back-calculation technique. Incidence is described and extrapolated through age-drift model; the observed survival is used for describing the patient prognosis. The survival is estimated through life-table method with moving window approach, using logistic regression model for future projections. The principal distinction between PIAMOD and our model is therefore a different approach for estimation of survival. The fully parametric representation of patient survival in PIAMOD model may be more suitable notably for projections in cancer diagnoses with good chance for long-term survival (e.g. Hodgkin disease, cancer of testis, etc).

Predictive population modelling allows us to work with different scenarios for individual epidemiological characteristics, which logically leads to different estimates of incidence and particularly prevalence. An example might be the trend in cancer incidence, which can either follow a particular trend, or it may show stabilized values (of course, expressed per 100,000 people). Therefore, the incidence rates can be modelled using statistical tools, or we can assume a stabilized behavior according to the latest available data. Another example of using scenarios in population-based modelling is the evaluation of cancer survival. Again, we can assume that survival rates are not changing over time, and calculate them using the most recent patient data.
On the other hand, we can assume that the improvement in cancer survival will show recently identified trends also in the future. In accordance with other epidemiological studies, for example [21], four scenarios combining progress in incidence and survival rates were considered in our prevalence model. However, results corresponding to only one of the scenarios are presented in this paper due to space restrictions.

In colorectal cancer, for example, we feel that the most likely scenario for the forthcoming years is the one with improving survival rates [10]; however, the stage-specific trends in incidence are not uniform. The incidence of stage I colorectal cancer is increasing due to earlier detection within colorectal cancer screening, where uptake of the screening program has substantially 
Tab. 9. Stage-specific estimates of prevalence of patients requiring active anti-tumor therapy for prostate cancer in the Czech Republic in 2015 and 2020. Numbers of patients are accompanied with 90\% confidence intervals (in brackets).

\begin{tabular}{|c|c|c|c|}
\hline Prostate (C61) & $\begin{array}{l}\text { Newly diagnosed cancer } \\
\text { patients }\end{array}$ & $\begin{array}{c}\text { Non-terminal recurrence } \\
\text { in patients from } \\
\text { previous years }\end{array}$ & $\begin{array}{l}\text { Terminal recurrence } \\
\text { in patients from } \\
\text { previous years }\end{array}$ \\
\hline stage I + II & $6,058(5,683 ; 6,434)$ & $4,785(4,671 ; 4,899)$ & \\
\hline stage III & $1,214(1,106 ; 1,321)$ & $1,536(1,472 ; 1,600)$ & \\
\hline stage IV & $874(820 ; 929)$ & $827(780 ; 874)$ & $1,176(1,120 ; 1,232)^{*}$ \\
\hline stage unknown & $51(24 ; 78)$ & $78(63 ; 93)$ & \\
\hline $\begin{array}{l}\text { number of treated patients in given } \\
\text { category }\end{array}$ & $\begin{array}{c}8,197 \\
(7,633 ; 8,762)\end{array}$ & $\begin{array}{c}7,226 \\
(7,086 ; 7,366)\end{array}$ & $\begin{array}{c}1,176 \\
(1,120 ; 1,232)\end{array}$ \\
\hline $\begin{array}{l}\text { total number of treated patients } \\
\text { year } 2020\end{array}$ & & $16,599(16,387 ; 16,811)$ & \\
\hline stage I + II & $8,489(7,969 ; 9,007)$ & $7,677(7,533 ; 7,821)$ & \\
\hline stage III & $1,644(1,500 ; 1,788)$ & $2,354(2,274 ; 2,434)$ & \\
\hline stage IIII & $1,060(981 ; 1,138)$ & $1,084(1,030 ; 1,138)$ & $1,274(1,215 ; 1,333)^{*}$ \\
\hline stage unknown & $26(7 ; 46)$ & $44(33 ; 55)$ & \\
\hline $\begin{array}{l}\text { number of treated patients in given } \\
\text { category }\end{array}$ & $\begin{array}{c}11,219 \\
(10,457 ; 11,979)\end{array}$ & $\begin{array}{c}11,159 \\
(10,985 ; 11,333)\end{array}$ & $\begin{array}{c}1,274 \\
(1,215 ; 1,333)\end{array}$ \\
\hline total number of treated patients & & $23,652(23,399 ; 23,905)$ & \\
\hline
\end{tabular}

increased in recent years [22]. On the other hand, stabilized incidence rates can be expected for advanced colorectal cancer. The improvement in survival rates can be attributed to the establishment of the network of highly specialized Comprehensive Cancer Centers that took place in the Czech Republic in 2006 [2], and the introduction of molecular targeted therapy in recent years.

Due to various health care interventions, e.g. the above mentioned screening programs, the recent situation in cancer epidemiology differs from diagnosis to diagnosis. For example, the incidence of breast carcinoma in females is significantly increasing (growth index 2001-2011: 22.9\%), it is accompanied with continuous increase of cases diagnosed early (according to recent data, $75.3 \%$ of incident cases were diagnosed in stage l or II) [3]. These positive changes are due to increasing impact of the Czech national breast cancer screening program [23] which already reached more than $50 \%$ coverage of target female population (aged 45-69 years). Similarly, as a consequence of widely used PSA test, we can observe a growing incidence of early detected prostate cancer although no organized screening for this type of cancer exists in the Czech Republic.

The most appealing and most arguable components of the model are cancer recurrence rates of patients diagnosed in the past and living in the year of interest. The rationale behind the estimation of functions representing the non-terminal and terminal cancer recurrence rates is to use surrogate parameters, which introduces high requirements on the data quality of the population-based registry. Such high quality of population-based data cannot be fully guaranteed; however, we feel that the estimates coming from the population-based databases may be more appropriate in this type of modelling, as the estimates calculated from clinical or hospital data can lead to biased results due to non-representativeness of the underlying set of patients.

\section{Conclusions}

In this paper, we present the stage-specific predictions of cancer incidence and prevalence, and stageand region-specific predictions of the number of patients requiring active anti-tumor therapy for malignant tumors in the Czech Republic in 2015 and 2020. The analysis, which utilizes solely the population-based cancer registry data, documents a serious increase in cancer incidence and prevalence in the Czech Republic in years 2015 and 2020 when compared to the situation in 2011. Moreover, the stage- and region-specific predictions for colorectal, lung, female breast, and prostate cancer implies correspondingly increasing numbers 


\section{Region-specific predictions for year 2015}

number of patients

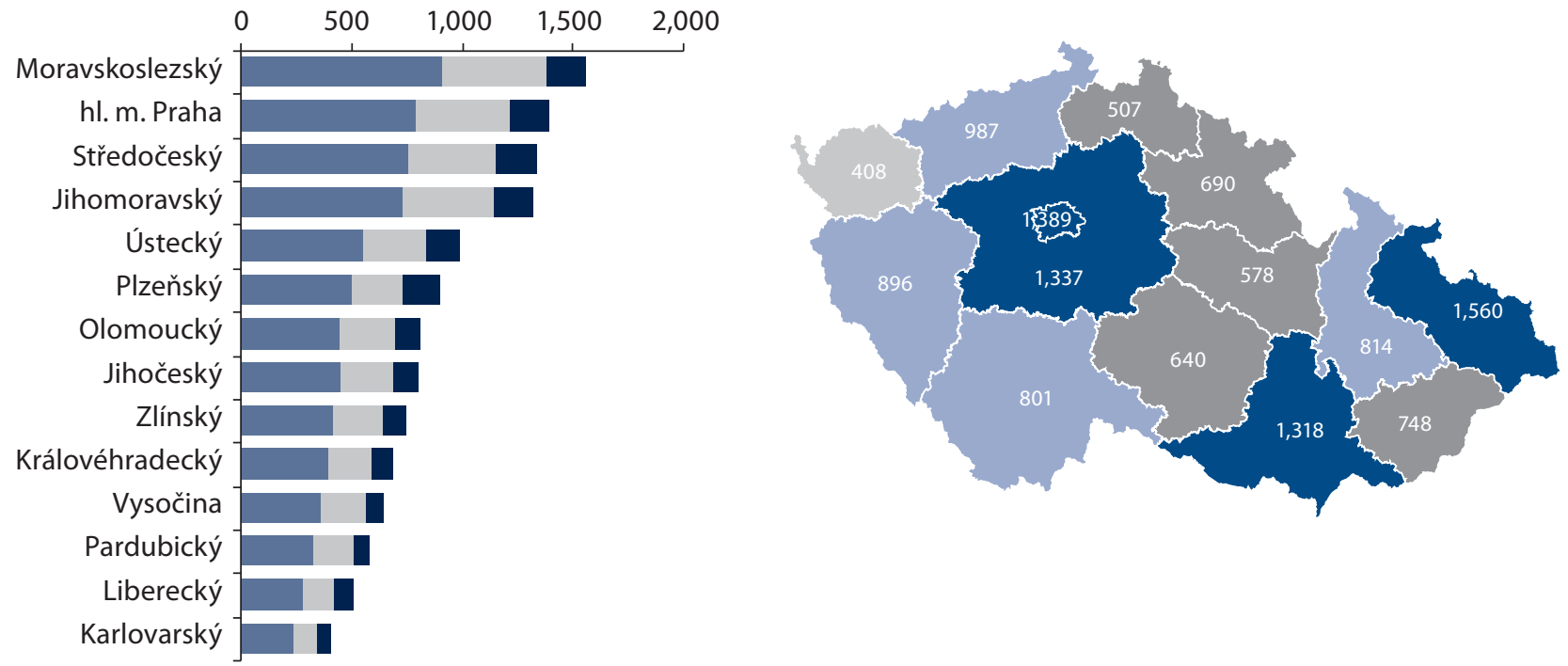

Region-specific predictions for year 2020

number of patients
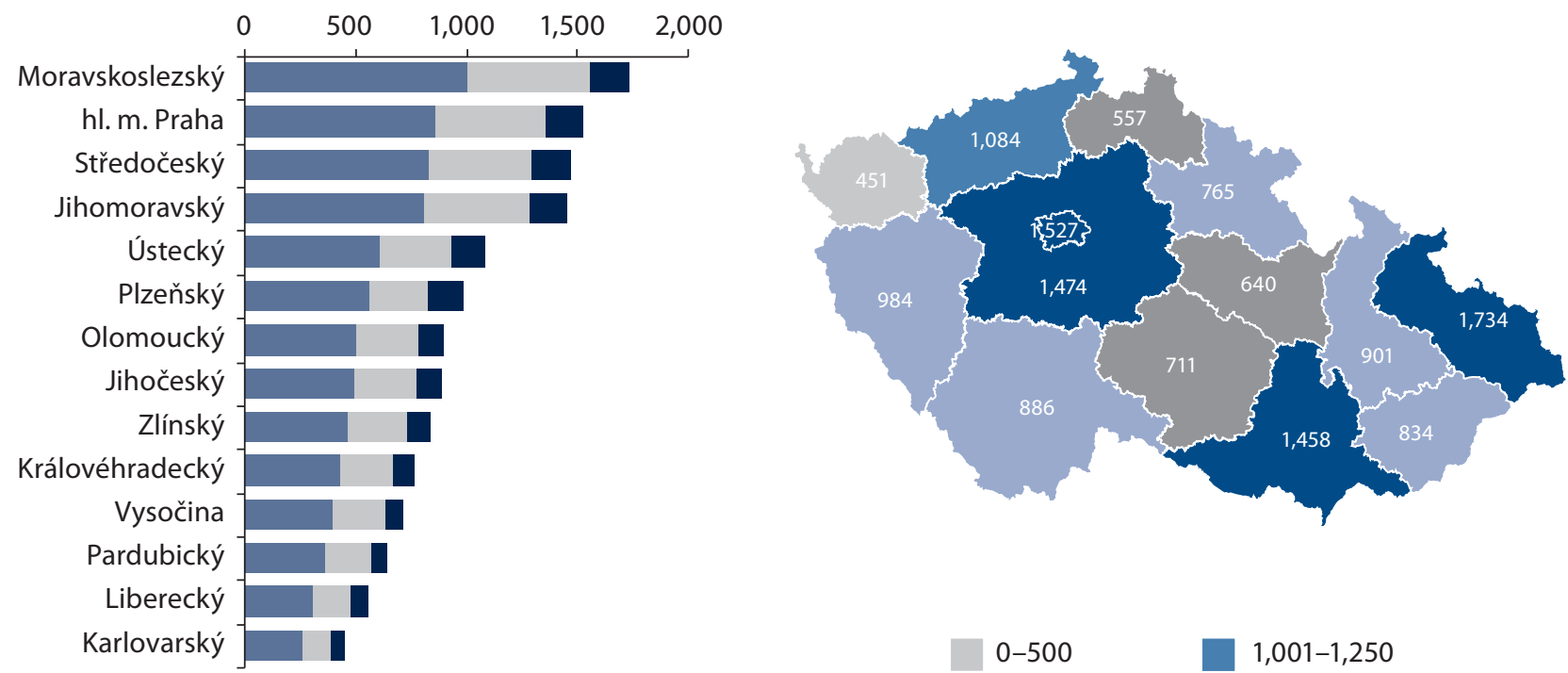

Pardubicky

Karlovarský

$1,001-1,250$

$501-750$

1,251 and more

newly diagnosed cancer patients

$751-1,000$

non-terminal relapses and progressions

terminal relapses and progressions

Fig. 2. Estimation of number of treated patients with colorectal cancer in Czech regions in 2015 and 2020.

of cancer patients treated in both the primary and the continuing care phase, therefore, underlying the increasing cancer burden in the Czech Republic in the following years.

\section{References}

1. Curado MP, Edwards B, Shin HR et al (eds). Cancer Incidence in Five Continents, Vol. IX. IARC Scientific Publications No. 160, Lyon, IARC, 2007 [cited 2013 May 8]. Available from: http://ci5.iarc.fr.

2. Dušek L (ed.). Czech Cancer Care in Numbers 2008-2009. Prague: Grada Publishing 2009.
3. Dušek L, Mužik J, Kubásek M et al. Epidemiology of malignant tumors in the Czech Republic [online]. Brno: Masaryk University; 2005 [cited 2014 May 8]. Available from: http://www.svod.cz. 4. Ferlay J, Steliarova-Foucher E, Lortet-Tieulent J et al. Cancer incidence and mortality patterns in Europe: estimates for 40 countries in 2012. Eur J Cancer 2013; 49(6): 1374-1403. doi: 10.1016/j.ejca.2012.12.027. 
5. Ferlay J, Soerjomataram I, Ervik M et al. GLOBOCAN 2012 v1.0, Cancer Incidence and Mortality Worldwide: IARC CancerBase No. 11 [Internet]. Lyon: International Agency for Research on Cancer; 2013. Available from: http://globocan.iarc.fr.

6. Boyle P, Ferlay J. Cancer incidence and mortality in Europe, 2004. Ann Oncol 2005; 16(3): 481-488.

7. Capocaccia R, Colonna M, Corazziari I et al. Measuring prevalence in Europe: the EUROPREVAL project. Ann Oncol 2002; 13(6): 831-839.

8. Clerc L, Jooste V, Lejeune $C$ et al. Cost of care of colorectal cancers according to health care patterns and stage at diagnosis in France. Eur J Health Econ 2008; 9(4): 361-367.

9. Gras C, Daurés JP, Tretarre B. Age and stage specific prevalence estimate of cancer from population based Cancer Registry using inhomogeneous Poisson process. Stat Methods Med Res 2004; 13(4): 273-289.

10. Pavlík T, Májek O, Mužík J et al. Estimating the number of colorectal cancer patients treated with anti-tumour therapy in 2015: the analysis of the Czech National Cancer Registry. BMC Public Health 2012; 12: 117. doi: 10.1186/1471-2458-12-117.
11. World Health Organization. International statistica classification of diseases and related health problems, 10th revision (ICD-10). Geneva: World Health Organization 1992

12. World Health Organization. International classification of diseases for oncology (ICD-O, 3rd revision). Geneva World Health Organization 2000

13. Sobin LH, Gospodarowicz MK, Wittekind Ch (eds). TNM classification of malignant tumors. 7th ed. Oxford: Wiley-Blackwell 2009

14. Hermanek P, Sobin LH (eds). International Union Against Cancer (UICC): TNM classification of malignant tumors. 4th edition. Berlin: Springer 1992.

15. Chambers JM, Hastie TJ (eds). Statistical models in S. Boca Raton: CRC Press 1991

16. Berrino $F$, De Angelis $R$, Sant $M$ et al. Survival for eight major cancers and all cancers combined for European adults diagnosed in 1995-99: results of the EUROCARE-4 study. Lancet Oncol 2007; 8(9): 773-783.

17. Yabroff KR, Mariotto AB, Feuer E et al. Projections of the costs associated with colorectal cancer care in the United States, 2000-2020. Health Econ 2008; 17(8): 947-959.
18. Maddams J, Utley M, Moller H. Projections of cancer prevalence in the United Kingdom, 2010-2040. Br J Cancer 2012; 107(7): 1195-1202. doi: 10.1038/bjc.2012.366. 19. Mariotto $A B$, Yabroff $K R$, Shao $Y$ et al. Projections of the cost of cancer care in the United States: 2010-2020. J Natl Cancer Inst. 2011; 103(2): 117-128. doi: 10.1093/jnci/dja495. 20. Verdecchia A, De Angelis G, Capocaccia R. Estimation and projections of cancer prevalence from cancer registry data. Stat Med 2002; 21(22): 3511-3526.

21. Gail MH, Kesser L, Midthune D et al. Two approaches for estimation disease prevalence from Population-based registries of incidence and total mortality. Biometrics 1999; 55(4): 1137-1144

22. Suchanek S, Majek O, Vojtechova G et al. Colorectal cancer prevention in the Czech Republic: time trends in performance indicators and current situation after 10 years of screening. Eur J Cancer Prev 2014; 23(1): 18-26. doi: 10.1097/CEJ.0b013e328364f203.

23. Majek O, Danes J, Skovajsova M et al. Breast cancer screening in the Czech Republic: time trends in performance indicators during the first seven years of the organised programme. BMC Public Health 2011; 11: 288. doi: 10.1186/1471-2458-11-288 